\title{
Kernos
}

Revue internationale et pluridisciplinaire de religion grecque antique

12| 1999

Varia

\section{Que représente la fresque de la paroi Ouest de la tombe au plongeur de Poseidonia?}

\section{Daisy Warland}

\section{OpenEdition \\ Journals}

\section{Édition électronique}

URL : http://journals.openedition.org/kernos/721

DOI : 10.4000/kernos.721

ISSN : 2034-7871

\section{Éditeur}

Centre international d'étude de la religion grecque antique

\section{Édition imprimée}

Date de publication : 1 janvier 1999

Pagination : 195-206

ISSN : 0776-3824

Référence électronique

Daisy Warland, «Que représente la fresque de la paroi Ouest de la tombe au plongeur de Poseidonia ? », Kernos [En ligne], 12 | 1999, mis en ligne le 13 avril 2011, consulté le 20 avril 2019. URL : http:// journals.openedition.org/kernos/721; DOI : 10.4000/kernos.721 


\section{Que représente la fresque de la paroi Ouest de la Tombe «du plongeur » de Poseidonia?}

En juin 1968, la découverte sensationnelle de la tombe « du Plongeur » couronna les campagnes de fouilles menées depuis de nombreuses années dans le golfe de Salerne ${ }^{1}$. Cette sépulture appartient à une nécropole implantée au lieu-dit "Tempa del Prete ", à moins de deux kilomètres au sud-est de Paestum, l'ancienne colonie grecque de Poseidonia. Du type à ciste orientée est-ouest, elle est constituée de cinq dalles de tuf peintes sur leur face interne de scènes figurées. Le style de ces dernières, à la transition entre l'art archaïque et l'art classique, confirme la datation de 480 av. J.-C. fournie par le lécythe attique à vernis noir faisant partie du mobilier funéraire.

Ce sont ces fresques qui constituent l'originalité et l'extraordinaire intérêt de la découverte puisqu'elle demeure jusqu'à ce jour unique - les rares tombes peintes $\mathrm{du}^{\mathrm{e}}$ siècle qui nous sont connues dans le monde grec ne portent pas de scènes figurées - et, partant, le seul monument qui nous évoque à sa juste échelle la « grande peinture », jusqu'alors abordée uniquement par le biais de la céramique décorée. Cet aspect ayant fait couler beaucoup d'encre, ce n'est pas lui qui va nous retenir ici, mais l'examen de l'iconographie, en particulier celle de la paroi Ouest ${ }^{2}$.

Tout d'abord, il faut souligner que cette unicité, qui ne peut être attribuée à des causes taphonomiques au vu de l'intensité des campagnes de fouilles menées dans la chora de Poseidonia, est un premier indice de la valeur cruciale de ces images. Leur conservation optimale a été expressément programmée, non seulement grâce à la qualité des pigments et des liants mis en

1 La première signalisation de la découverte fut communiquée dans : M. NaPoli, L'attività arcbeologica nelle provincie di Avellino, Benevento e Salemo, in La Magna Grecia e Roma nell'età arcaica. Alti dell'ottavo Convegno di Studi sulla Magna Grecia. Taranto, 6-11 otlobre 1968, Naples, 1969, p. 139-152. Il affina son analyse dans: M. Napoli, Le pitture greche della Tomba del Tuffatore, in Le Scienze, 2, 8 (1969), p. 9-19; et publia un ouvrage entièrement consacré à l'étude de la tombe : M. NAPoli, La Tomba del Tuffatore. La scoperta della grande pittura greca, Bari, 1970.

2 Une analyse iconologique générale, dont on rappelle ici quelques résultats - de façon un peu abrupte - en ce qui concerne les autres parois, fait le sujet de : D. WARLAND, Tentative d'exégèse des fresques de la tombe "du Plongeur " de Poseidonia, in Latomus, 57 (1998), p. 261-291. Voir aussi D. WArland, La tombe " du Plongeur». Étude de la relation entre le symposion et le plongeon, in $R H R, 213-2$ (1996), p. 143-160. 
œuvre ${ }^{3}$, mais aussi par la réalisation particulièrement soignée et le cimentage exceptionnel des joints entre les dalles. Comme le prouvent les traces de corde marquées dans la peinture, elles ont été réalisées avant leur installation in situ, très probablement en atelier ${ }^{4}$ et en fonction de la personnalité du défunt. Peintes sur les parois internes de la tombe scellée, c'est bien à lui, et à lui seul, qu'elles s'adressent, en tant que rite funéraire à valeur magico-religieuse. Elles sont inséparables d'une croyance en une forme de survie après le trépas.

«Hapax dans l'bapax», la dalle de couverture révèle une scène de plongeon sans égal ${ }^{5}$, symbole du passage de la vie terrestre à la mort, transcendée dans la survie après régénération cathartique par les eaux marines. Les parois latérales présentent des scènes de symposion, thème de prédilection de la céramique attique à figures rouges d'après 520-510 av. J.- $\mathrm{C}_{1}^{6}$ et de la peinture funéraire étrusque ${ }^{7}$.

La paroi Nord combine les motifs du désir amoureux et du jeu du kottabos, exprimant lui aussi une recherche de faveurs ${ }^{8}$. En insistant sur l'atmosphère érotique déjà suggérée par la répétition des couples éraste-éromène,

3 Les fresques étaient dépourvues des incrustations calcaires et de la couche noire habituellement présentes dans les tombes peintes.

4 Selon une étude minutieuse de la technique des fresques - et de leur restauration -, ces cordes auraient servi non pas à positionner les dalles in situ mais à les manipuler en atelier, puisque la peinture était sèche, comme en témoigne un repentir réalisé à l'argile: A. Melucco Vaccaro, in Il dibattito, in Poseldonia-Paestum. Atti del ventisettesimo Convegno di Studi sulla Magna Grecia. Taranto, 9-15 ottobre 1987, I, Naples, 1988, p. 338-357, particulièrement p. 356.

5 Outre la tombe « du Plongeur», les seules monuments antiques à nous avoir transmis des images de plongeon en action sont : la tombe « de la Chasse et de la Pêche » de Tarquinia (S. SteingräBer [éd.], Catalogo ragionato della pittura etrusca, Milan, 1984 [ultérieurment noté « S »], 50; S. DE MARINIS, La tipologia del bancbetto nell'arte etrusca arcaica, Rome, 1961 [ultérieurement noté « DM »], 36; ca. 510 av. J.-C.) et le cratère apulien en calice à figures rouges dit « du Soleil et des Heures» du British Museum (C.H. SMITH, Catalog of the Greek and Etruscan Vases in the British Museum, III, Vases of the Finest Period, Londres, 1896, p. 284-285 [ca. 480 av. J.-C.]; LIMC, II, 1, s.v. Astra [S. Karusu], p. 910-911, fig. 22; LIMC, III, 1, s.v. Endymion [H. GabelmanN], p. 729 et III, 2, p. 552, fig. 11 [ca. 420 av. J.-C.]). Elles appartiennent au domaine italien. Dans les deux cas, il s'agit d'un jeune homme nu se précipitant la tête la première dans la mer, mais en position verticale et entouré de nombreux autres personnages.

6 Le thème du banquet dans l'art grec a été étudié par B. FEHR, Orientaliscbe und griechische Gelage, Bonn, 1971; J.-M. Dentzer, Le motif du banquet coucbé dans le Procbe-Orient et le monde grec du VII au IVe siècle av. J.C., Rome, 1982 (BEFAR, 246); R. HurischmanN, Symposienszenen auf unteritalischen Vasen, Wurzbourg, 1985.

7 Dont un répertoire figure dans De Marinis, op. cit. (n. 5). D'autres occurrences se trouvent dans le catalogue plus récent de StreingräBER, op. cit. (n. 5).

8 Cf. Daremberg-Saglio-Pottier, III, 1 (rééd. 1969), s.v. Kottabos; F. Lissarrague, un flot d'images. Une estbétique du banquet grec, Paris, 1987, p. 80. 
ces deux scènes évoquent l'initiation sexuelle dans les sociétés d'hommes archaïques $^{9}$ et, plus généralement, la puissance vitale.

La paroi Sud nous présente successivement les thèmes de l'extase musicale, signe d'entbousiasme et non d'ivresse ou de chant ${ }^{10}$, de la conversation, activité pouvant déboucher sur la discussion philosophique ${ }^{11}$, et d'un banqueteur tenant une lyre et un œuf, « la musique étant cette méta-érotique dont la fonction essentielle est à la fois de concilier les contraires et de maîtriser la fuite existentielle du temps $»^{12}$. L'œuf, offrande funéraire omniprésente $^{13}$, symbolise non seulement la fécondité, mais plus encore la puissance vitale dans la mort et la résurrection ${ }^{14}$.

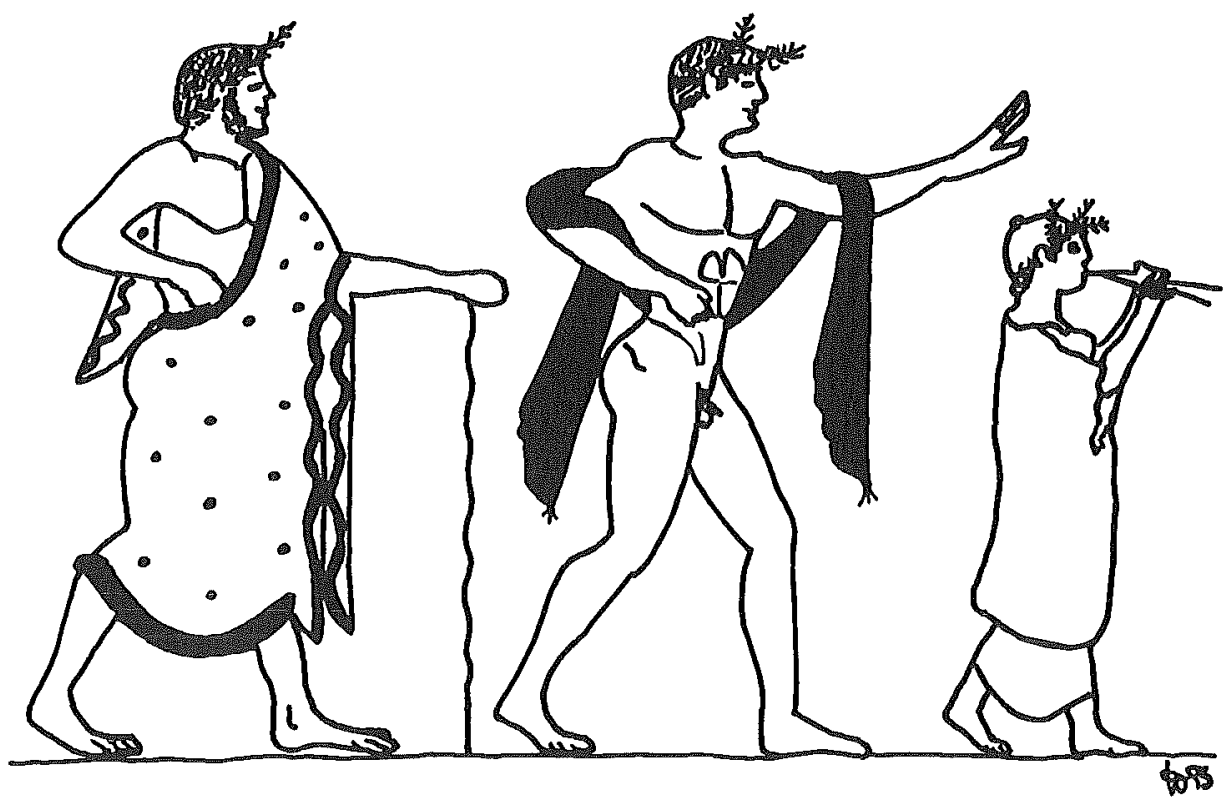

Fig. 1 : Paroi Ouest de la tombe «du Plongeur » de Poseidonia (480 av. J.-C.)

[dessin de l'auteur d'après photo].

9 Cf. B. Sergent, L'bomosexualité dans la mythologie grecque, Paris, 1981.

10 Gf. P. Somville, Le signe d'extase et la musique, in Kernos, 5 (1992), p. 173-181.

11 Cf. Platon, Le Banquet.

12 G. DURAND, Les structures antbropologiques de l'imaginaire. Introduction à l'arcbétypologie générale, Paris, $1984^{10}$, p. 400.

13 Cf. M. Astruc, La necropolis de Vilaricos, Madrid, 1951, p. 96-101.

14 Comme encore de nos jours les " oeufs de pâques ». Cf. Durand, op. cit. (n. 12), p. 289-290. On trouvera une profusion de comparaisons ethnologiques dans M. Eliade, "L'œuf cosmogonique", in Traité d'bistoire des religions, Paris, 1989, p. 347-349. 
La paroi Est représente un éphèbe-échanson tenant une onochoé, qui nous ramène dans la sphère des initiations masculines ${ }^{15}$, et un cratère, signe matériel de la mesure du vin ${ }^{16}$, du mélange et du partage. Appartenant au domaine des contenants, il « se situe à mi-chemin entre les images du ventre digestif ou sexuel et celles du liquide nutritif, de l'élixir de vie et de jouvence $\gg^{17}$.

La compréhension de la scène figurant sur la paroi Ouest est moins immédiate. Les trois personnages sont les seuls du cycle à aller dans la direction opposée au sens de lecture ${ }^{18}$. Regardant droit devant eux, ils avancent vers la droite d'un même pas, jambes légèrement fléchies, la gauche en avant, talon au sol, et la droite en arrière, talon soulevé. Par commodité, je les désignerai par la lettre $O$, signalant qu'il s'agit de la plaque Ouest, suivie d'un chiffre indiquant leur position ( 1 à 3 de droite à gauche).

O1 est la seule femme du cycle. Elle est de petite taille et ses chairs, non peintes, prennent la couleur de la paroi de tuf blanc. La carnation claire est caractéristique de la représentation féminine dans la peinture grecque, étrusque et lucanienne (déjà minoenne et égyptienne). Elle porte un chiton blanc étroit descendant jusqu'à la cheville, recouvert d'une tunique plus ample s'arrêtant sous le genou et laissant les avant-bras nus. Elle est coiffée d'une calotte rouge, ceinte d'une couronne particulière: les feuilles se concentrent à l'avant, un rameau brisé penche et un troisième rameau pointe

15 Sur le modèle mythique de Ganymède, enlevé par Zeus.

16 Le vin n'était jamais bu pur, mais mélangé à $2 / 3$ à $3 / 5$ d'eau froide ou tiède. La mesure est une préoccupation essentielle des Grecs. La mythologie souligne incessamment le caractère criminel de l'bybris.

17 Durand, op. cit. (n. 12), p. 293.

18 Il faut adopter un sens de lecture droite-gauche. Les personnages couchés sont pratiquement toujours orientés dans ce sens, autant dans l'art grec qu'étrusque. Ils s'appuient sur le bras gauche replié, le coude reposant sur un coussin, afin de libérer le bras droit. Ils sont par conséquent tournés du côté gauche. Pour les montrer de face, le peintre était obligé de choisir ce sens. Dans les cas exceptionnels où le convive est montré de dos, la même orientation apparaît; il est donc appuyé sur son coude droit. Les scènes de prothesis représentent le défunt couché la tête à droite, depuis les vases grecs de l'époque géométrique jusqu'aux tombes lucaniennes. Or, les banqueteurs changeaient souvent de position au cours de leurs interminables agapes, et d'un point de vue réaliste, rien n'empêchait l'artiste de les représenter appuyés sur le coude droit, couchés sur le dos ou sur le ventre. C'est donc le sens de lecture qui dicte la position des personnages et non le contraire; c'est lui qui est signifiant. Même s'il est devenu une simple convention de composition artistique, il est probable qu'à l'origine, il répond à la symbolique fondamentale de la définition de l'espace par rapport à l'homme. La droite étant bénéfique et la gauche maléfique, il fallait absolument placer la tête à droite et les pieds à gauche. L'iconographie du banquet montre qu'on porte la coupe à ses lèvres de la main droite, qu'on joue au kottabos à la main droite, et qu'on sert le vin avec une œenochoè tenue dans la main droite et en versant de droite à gauche (servir à boire à quelqu'un en versant de gauche à droite est encore considéré de nos jours en Italie comme portant malheur). 
au-dessus de l'oreille. Elle souffle dans un aulos ${ }^{19}$, tenu tuyaux parallèles, légèrement inclinés vers le bas. Les aulétrides, majoritaires dans la céramique grecque, sont au contraire pratiquement absentes de l'art étrusque ${ }^{20}$.

$\mathrm{O} 2$ est un éphèbe, imberbe, qui porte pour tout vêtement une chlamyde bleue en étole. Sa couronne est à deux rameaux un peu incurvés : le premier, brisé, descend au-dessus du front et le second se dresse au-dessus de l'oreille. La tête et les épaules sont légèrement projetées vers l'avant. Le bras gauche est tendu vers la droite si bien que la main arrive au-dessus de la tête de O1. Le bras droit est plié, coude vers l'arrière.

O3 est un homme drapé dans un long manteau blanc, bordé de rouge et semé de pois, qui lui descend sous le genou et dont un pan est rejeté par dessus son épaule gauche. Son attitude est proche de celle de $\mathrm{O} 2$, mais il a le buste droit et l'avant-bras gauche tendu horizontalement à hauteur de la taille pour s'appuyer sur un long bâton noueux, planté verticalement devant lui. Cet objet, marque d'une recherche d'élégance ${ }^{21}$, apparaît fréquemment sur les vases attiques, le plus souvent dans la main d'adultes ${ }^{22}$.

L'interprétation de cette scène est controversée. Que représente-t-elle exactement? Une des premières hypothèses venue à l'esprit des chercheurs est celle de l'entraînement sportif ${ }^{23}$ ou du début d'un concours ${ }^{24}$. Or, comme le relève $\mathrm{Napoli}^{25}$, elle répond au décryptage de la scène du plongeon dans le même sens, ce qui ne fait que déplacer le problème. Il est vrai que la Grande Grèce s'est brillamment illustrée dans les concours. Les vainqueurs étaient honorés. Les tombes d'athlètes, retrouvées notamment à Tarente, ne ressemblent cependant en rien à la tombe "du Plongeur ". Elles exposent les vases remis au champion comme prix de sa victoire ${ }^{26}$. Quoi qu'il en soit, les sources littéraires ne font pas état du plongeon comme d'un sport. Il est certain qu'il ne figurait pas au programme des concours. On sait que des indivi-

19 Il est généralement appelé «flûte », voire « double-flûte », par les auteurs modernes. Cette traduction est erronée, étant donné qu'il comporte une anche (simple ou double). $C f$. l'excellente étude organologique de K. Schlesinger, The Greek Aulos, Londres, 1939.

20 À l'exception du sarcophage du Museo Civico de Chiusi (DM 76) et de la tombe " des Biges » ou « Stackelberg » de Tarquinia (S 47, DM 41, ca. 490 av. J.-C.).

21 LAMER, s.u. kômos, in RE, XI 2 (1922), col. 1294.

22 Par ex. kylix attique à figures rouges du Kunsthistorisches Museum de Vienne: CVA, Österreich 14, Wien 1, pl. 14 (début ve siècle av. J.-C.).

23 Comme le suggérait Lo Porto à Napoli : $c f$. M. NAPoli, L'attività arcbeologica nelle provincie di Avellino, Benevento e Salemo, in La Magna Grecia e Roma nell'età arcaica. Atti del VIII Convegno di studi sulla Magna Grecia, Taranto, Naples, 1969, p. 139-152, particulièrement p. 149.

24 B. Bilinski, in Il dibattito, ibidem, p. 265.

25 Napoli, loc. cit. (n. 23), p. 149-150 et op. cit. (n. 1), p. 145.

26 F.G. Lo Ponto, Tombe di atleti tarentini, in $A S M G, 8$ (1967), p. 69 sq. 
dus se sont jetés dans la mer du haut de falaises, mais il s'agit toujours de faits exceptionnels fortement significatifs, à caractère mythologique ou rituel, et pas d'activités institutionnalisées, à deux exceptions près : le saut de Leucade, en rapport avec le culte d'Apollon ${ }^{27}$, et le concours d'Hermionè, dans le cadre du culte de Dionysos Melanaigis - pour autant qu'il s'agisse effectivement de plongeon ${ }^{28}$.

Enfin, si l'on compare le motif de la paroi Ouest avec la peinture attique de vases à figures rouges, on trouve effectivement plusieurs scènes de palestre très proches, mais elles diffèrent par deux détails importants. D'abord, c'est toujours un aulète qui rythme l'exercice. O1 est une femme, qui n'aurait pas sa place dans le monde agonistique exclusivement masculin. Ensuite, les jeunes athlètes nus et leurs maîtres barbus en manteau tiennent toujours en main des haltères, des strigyles ou des javelots ${ }^{29}$. Ainsi, il apparaît clairement que la paroi Ouest est dépourvue de connotation sportive.

La seconde hypothèse envisagée tourne autour du thème du cortège : kômos pour Rösler ${ }^{30}$, voyage vers l'au-delà pour Napoli ${ }^{31}$ et Somville ${ }^{32}$, schéma de répertoire sans valeur symbolique pour Bianchi Bandinelli ${ }^{33}$.

Le kômos appartient à la sphère symposiaque. Lorsque le banquet était terminé, les convives qui ne rentraient pas directement chez eux s'exhibaient en rue dans des cortèges débridés, dansant, chantant et jouant de la musique. L'origine de cette coutume est à rechercher dans les processions rituelles intégrées au culte de Dionysos. Ce type de scène est très fréquent dans la céramique grecque et la peinture étrusque. Il est viai que les comastes sont généralement plus nombreux, qu'ils tiennent souvent des vases à boire

27 Strabon, X, 2, 9.

28 La seule compétition (agôn) de plongeon (kolumbos) attestée serait celle, annuelle, d'Hermioné, près de Trézène, au temple de Dionysos Melanaigis, à l'égide noire, associée à un concours de musique et de course de bateaux (Pausanias, II, 35, 1). Le terme ambigu de kolumbos ne permet pas de trancher s'il s'agit réellement de plongeon ou de natation. Cependant, c'est le nom du Prodiceps minor, canard dont le nom vulgaire français est «plongeon» et dont l'appellation latine antique était mergus (cf. Ovide, Métam., XI, 749795). Le même terme de kolumbos est employé dans le fragment 94 d'Anacréon faisant allusion au saut de Leucade. Enfin, les jeux d'Hermioné célèbrent Dionysos, dont les mythes contiennent plusieurs occurrences de plongeons (cf. HoM., Il., VI, 130-140; Hymne pseudo-bomérique à Dionysos, 52-54; Pausanias, II, 37, 5-6).

29 Par ex. coupe attique à figures rouges de Douris de l'Antikenmuseum de Bâle: CVA, Suisse 6, Bâle 2, pl. 19, 2-4 et 20, 1-2 (ca. 490 av. J.-C.).

30 W. Rösler, Eine Komos-Darstellung im 'Grab des Tauchers' in Paestum?, in $A A$ (1983), p. 13-15.

31 NAPOL, loc. cit. (n. 23), p. 150 et op. cit. (n. 1), p. 145-146.

32 P. Somville, La tombe du plongeur à Paestum, in Études grecques, Bruxelles-Liège, 1990 , p. 25-33, particulièrement p. 26.

33 R. BiANChi BANDINELli, DdA, 4 (1970-1971), p. 135-142, particulièrement p. 141. 
(kylikes ou skyphoi), et que leur attitude est moins tranquille, voire exubérante ${ }^{34}$.

La réduction du cortège à un minimum de deux personnes, l'aulétride et un éphèbe, est attestée ${ }^{35}$. On trouve également des kômoi de trois personnes, à savoir une aulétride et deux éphèbes ${ }^{36}$. Les comastes sont parfois couronnés ${ }^{37}$. La peinture funéraire étrusque présente de nombreux exemples de cortèges de jeunes danseurs et danseuses exubérants, accompagnés de l'aulos ou de la lyre ${ }^{38}$.

La paroi Ouest de la tombe « du Plongeur » présenterait une combinaison originale des éléments du kômos. L'attitude de l'éphèbe est semblable à celle des danseuses représentées sur les métopes archaïques de l'Héraïon de l'embouchure du Sele ${ }^{39}$. Il est donc vraisemblable, comme le suggèrent entre autres Napoli ${ }^{40}$ et Rösler ${ }^{41}$, que l'éphèbe esquisse un pas de danse. (On aurait ici la transmission d'un schéma archaïque pour l'expression de ce mouvement).

La chlamyde jetée autour des bras et le « pas de danse » de l'éphèbe, les couronnes, la présence de l'aulétride permettent de confirmer l'hypothèse du

34 Ex. proches du kômos de la paroi Ouest : une pélikè attique à figures rouges du Museum Antiker Kleinkunst de Munich (CVA, Deutscbland 6, München 2, pl. 70, ca. 470 av. J.-C.) et une kylix de style attique à figures rouges du Museo Civico de Bologne (CVA, Italia 33, Bologne 5, pl. 116, ca. 460-450) montrent un homme barbu en manteau et un éphèbe nu avec la chlamyde sur l'épaule encadrant respectivement une aulétride et un aulète, le cortège continuant sur l'autre face, avec trois autres personnages.

35 Par ex. cratère campaniforme italiote à figures rouges du Museo Nazionale de Tarente : CVA, Italia 35, Taranto 3, pl. 9.

36 Par ex. cratère en cloche italiote à figures rouges des Musei Capitolini de Rome : CVA, Italia 39, Rome 2, pl. 24 ( $c a .440-430$ av. J.-C.). les éphèbes ont une chlamyde sur l'épaule et sont appuyés sur des bâtons.

37 Par ex. stamnos de style attique à figures rouges du peintre d'Hermonax, du Louvre : CVA, France 4, Louvre 3, pl. 12, 3-6.

38 Par ex. tombe « des Biges » ou «Stackelberg » de Tarquinia (S 47, DM 41, ca. 490 av. J.-C.). Trois femmes en robe et épibléma, dont une joue de l'aulos, dansent avec trois éphèbes, le manteau noué en pagne, avec de grands rameaux entre les personnages. Une scène de symposion continue sur la paroi successive. La présence de danseuses féminines n'est pas une objection à l'identification d'un kômos, puisqu'en Étrurie, les maîtresses de maison prenaient part au banquet, aux côtés de leur époux. - La tombe « de la Colline » ou «Casuccini » de Chiusi $(S 15, D M 50$, deuxième quart du v siècle av. J.-C.) présente un cortège de seuls éphèbes, chlamyde jetée sur les épaules, dont un joue de l'aulos et le dernier à gauche, en manteau, de la lyre. Des branchages ou arbrisseaux les séparent, comme dans l'exemple précédent.

39 Cf. P. Zancani Montuoro et U. Zanotti-Bianco, Heraion alla foce del sele, Rome, 1951, I, p. 121-161 et II, pl. XLI-LIX. Ces métopes sculptées représentent un choros liturgique de jeunes filles, d'une grande souplesse et vivacité narrative.

40 Napoli, op. cit. (n. 25), p. 144, remarque que l'éphèbe «avance d'un pas léger, quasi rythmé, comme accompagné de la double flûte ».

41 RösLER, loc. cit. (n. 30), p. 14. 
kômos comme étant la meilleure, d'autant plus qu'elle a le mérite d'intégrer la paroi au programme des trois autres.

$\mathrm{Au}$ bras tendu vers l'avant de $\mathrm{O} 2$ répond visuellement le geste du cinquième banqueteur de la paroi Nord, comme si, plutôt que cible du jeu de kottabos, sa coupe était levée pour saluer l'éphèbe, ce qui confirme le rattachement du cortège aux scènes de banquet.

Le kômos serait formé de deux personnes arrivant d'un autre symposion pour se joindre aux banqueteurs présents ou quittant le symposion en cours, précédés de la jeune aulétride ${ }^{42}$. Ces deux convives supplémentaires pourraient expliquer les deux places vacantes sur les klinai de gauche des parois Nord et Sud, mais on attendrait plutôt deux jeunes gens, pour respecter le schéma d'occupation des lits en couple éraste-éromène. Vraisemblablement, ils forment eux-mêmes un autre couple de ce type. La singularisation de leurs couronnes, montrant une gradation de un à trois rameaux, est un autre argument en faveur de la première solution.

Une fois adoptée l'interprétation du kômos, l'analyse à un niveau sémantique supérieur requiert d'autres investigations.

Pour justifier son hypothèse d'une image de l'abandon de la vie terrestre, Napoli avance deux arguments. Le premier, interne, est que le cortège marche dans le sens opposé au sens de lecture, comme s'il quittait le symposion. Or, cela équivaudrait, semble-t-il, à réduire ce dernier à l'illustration de la vie sur terre. En réalité, les différentes interprétations que l'on peut donner aux scènes de banquet ne sont que les facettes d'une même volonté de garantir une heureuse survie dans l'au-delà : perpétuation du rituel d'offrandes alimentaires au défunt, rappel des expériences dionysiaques supra-naturelles qui rapprochent les hommes des dieux durant leur vie terrestre, festin des bienheureux dans leur vie éternelle, atmosphère érotique évoquant la puissance vitale. Le second argument invoqué par Napoli, externe cette fois, est que les tombes lucaniennes présentent souvent une scène de voyage vers l'au-delà sur leur côté Ouest, comme le départ d'un cavalier, salué par sa femme et suivi d'un homme en manteau. En fait, cette image est habituellement interprétée comme le retour du guerrier, et est aussi souvent présente sur la paroi Est que sur la paroi Ouest. Les deux seules scènes qui illustrent sans aucune ambiguité possible le voyage vers l'au-delà, à savoir la défunte montant dans une barque menée par une démone ailée à mi-chemin entre la Vanth étrusque et le Charon romain dans la tombe $\mathrm{n}^{\circ} 47 \mathrm{~A}$, et Hermès tirant un chariot où est assis un enfant dans la tombe $\mathrm{n}^{\circ} 8 \mathrm{~A}$, sont peintes sur la

42 Cf. Platon, Banquet, 176 e et $212 \mathrm{~d}$ : une fois le repas terminé, l'aulétride doit sortir, afin de ne pas déranger le symposion, exceptionnellement voué aux discours philosophiques, et précéder ceux qui quittent la salle. Une autre apparaît pour escorter Alcibiade : il s'agit d'un kômos sorti d'un autre symposion qui vient se terminer chez Agathon. 
paroi Est $^{43}$. Le thème du voyage vers l'au-delà sous sa forme terrestre, à cheval ou en charrette, est par ailleurs amplement illustré dans l'art étrusque ${ }^{44}$.

Ne pourrait-on envisager, au contraire, que le cortège illustrerait l'intégration du défunt à sa nouvelle vie, après sa régénération par la mer, figurée sur le couvercle? Cette interprétation originale m'a été suggérée par la lecture du livre XV des Métamorphoses ${ }^{45}$, narrant l'épisode de la résurrection d'Hippolyte ${ }^{46}$.

Les parallèles, quoiqu'anachroniques, entre les vers d'Ovide et les images peintes dans la tombe sont troublants. Comment ne pas penser à la scène du plongeon en lisant : " j'ai vu aussi le royaume des ténèbres, et j'ai cherché dans les eaux du Phlégéton la guérison pour mon corps mutilé $"{ }^{47}$ ? Où trouver meilleure description de la mer telle que l'ont représentée les artistes de Poseidonia, barrée par la grande cassure de la dalle : « et l'on vit une énorme masse d'eau s'arrondir et grossir à l'image d'une montagne. Elle pousse des mugissements et se fend à son sommet (...) ${ }^{48}$ ? Les deux arbres penchés qui enveloppent le katapontismos ne sont-ils pas ces plantes miraculeuses: «Quand, grâce aux vertus bienfaisantes de ses plantes (...) je l'eus recouvrée (la vie) $\gg{ }^{49}$ ?

43 Cf. A. Greco Pontrandolfo, A. Rouveret, Ideologia funeraria e società a Poseidonia nel IV sec. a.C, in G. GNoli et J.-P. Vernant (dir.), La mort, les morts dans les sociétés anciennes, Cambridge-Paris, 1982, p. 299-317; A. Greco Pontrandolfo, A. RouveRET, Pittura funeraria in Lucania e Campania. Puntualizzazioni cronologicbe e proposte di lettura, in DdA, n.s. III, 1 (1983-2), p. 91-130; A. Greco Pontrandolfo, A. Rouveret, Le tombe dipinte di Paestum, Modène, 1992 (catalogue p. 82-302); M. Roosens, La pittura funeraria antica nell'Italia meridionale (status quaestionis), thèse KUL, Louvain, 1989, p. 24-38; A. Rouveret, L'organisation spatiale des tombes de Paestum, in MEFRA, 87, 2 (1975), p. 595-652; D. WaRland, Étude de la composition des peintures de la tombe $n^{\circ} 47$ de Paestum, in $A C, 67$ (1998), p. 169-182.

44 Par ex. stèles de Bologne : A. Grenier, Bologne Villanovienne et Etrusque. VII ${ }^{e}-I^{e}$ slècles avant notre ère, Paris, 1912, p. 435-452. Autres ex. dans C.C. VAN EssEN, Did Orphic Influences on Etruscan Tomb Painting Exists? Studies in Etruscan Tomb Painting I, Amsterdam, 1927, p. 24-29.

45 Ovide, Métam., XV, 500-547 (trad. J. Chamonard, II, Classiques Garnier, Paris, 1953).

46 Asclépios, fils d'Apollon et de Coronis, princesse thessalienne, fut élevé par le centaure Chiron qui lui enseigna la médecine. Mais il abusa de son art en ressuscitant Hippolyte, fils de Thésée et d'Hippolitè (ou d'Antiope), mis à mort par Poséidon sur la demande de 'Thésée, suite à la dénonciation posthume de Phèdre, dont les ardeurs coupables, inspirées par Aphrodite à laquelle Hippolyte préférait Artémis, avaient été repoussées, Cf. aussi Chants de Naupacte, in Apollodore, III, 10, 3; Euripide, Hippolytos Kalyptomenos (19 fragments conservés) et Hippolytos Stepbanios (ou Stephanopboros); Hésiode, fr. 51; Pausanias, II, 27, 4; Pindare, Pytbiques, III, 45 et 54-60; Sophocle, Pbèdre (11 fragments conservés); Virgile, Énéide, VII, 761-784.

47 Ovide, Métam., XV, 531-532.

48 Id., 507-510.

49 Id. $534-535$. 
Ainsi, il est tentant d'assimiler $\mathrm{O} 3$ et $\mathrm{O} 2$ à des doublets d'Asclépios et d'Hippolyte ramené à la vie, et $\mathrm{O} 1$ à Hygie, la fille du dieu. On remarquera au passage que le thème du katapontismos est récurrent dans la mythologie de Thésée, le père d'Hippolyte, avec des connotations ordaliques, initiatiques et eschatologiques ${ }^{50}$.

L'iconographie de ces personnages est tardive mais permet quelques comparaisons $^{51}$. Asclépios est généralement représenté comme un adulte barbu, enveloppé dans un long manteau qui laisse son épaule droite découverte, et appuyé sur un bâton noueux ${ }^{52}$ autour duquel s'enroule un serpent ${ }^{53}$. Hippolyte apparaît sous les traits d'un éphèbe vêtu d'une chlamyde et portant une pique de chasseur. Sa mort est représentée uniquement sur des monuments italiotes et étrusques ${ }^{54}$.

Les origines du culte d'Asclépios sont mal connues. Il s'agissait probablement d'un héros guérisseur de Tricca en Thessalie ${ }^{55}$, progressivement honoré dans toute la Grèce, notamment à Épidaure dès le vi ${ }^{\mathrm{e}}$ siècle av. J.-C. et à Athènes à la fin du v $\mathrm{v}^{\mathrm{e}}$ siècle av. J.-C., avant d'atteindre Rome en 291 av. J.-C. Sa présence à Paestum est attestée par Ovide : ayant traversé la mer ionienne, Asclépios longe les côtes italiennes, en passant par « les champs de roses de la tiède Paestum ${ }^{56}$. Mais aucune trace archéologique de son culte ne nous est parvenue, si ce n'est peut-être un cippe trouvé devant le temple d'Héra I portant l'inscription Cbirônos $^{57}$. La légende d'Hippolyte aurait pris naissance à Trézène ${ }^{58}$. Les colons l'auraient introduit à Aricie, où il se serait associé à Virbius, génie local de la forêt ${ }^{59}$. Or, Poseidonia aurait été fondée vers 600 av. J.-C. par des Grecs d'origine thessalo-béotienne ${ }^{60}$ ou à la fin du viII ${ }^{\mathrm{e}}$ siècle par

50 Cf. le défi de Minos dans Bacchylide, Ode XVII; la mort de Thésée dans Plutarque, Vie de Thésée, 35, 6 et 7; l'exécution de Skiron dans Pausanias, I, 44, 8.

51 Cf. LIMC, II, 1 (1984), s.v. Asklepios (B. Holtzmann), p. 863-897 et LIMC, V, 1 (1990), s.v. Hippolytos I (P. Linant De Bellefonds), p. 445-464.

52 Le bâton est parfois tenu à bout de bras : par ex. statue $\mathrm{n}^{\circ} 8645$ du Museo Nazionale de Rome, provenant du Quirinal (LIMC, V, 2 [1990], p. 877, fig. 116).

53 Le serpent est parfois absent : par ex. relief $n^{\circ} 462$ de la Ny Carlsberg Glyptotek de Copenhague, provenant du Pirée (LIMC, V, 2 [1990], p. 881, fig. 211).

54 Cf. LIMC, V, 1, p. 458 et 460.

55 STrabon, IX, 5, 17.

56 OvIDE, Métam., XV, 708.

57 Cf. M. Guarducci, Paestum; Cippo arcaico col nome di Chirone, in Not. Sc., 1948, p. 185 sq.

58 Eur., Hipp., 1423-1425; PAUS., II, 32, 1.

59 Par ex. paus., II, 27, 4. Cf. L. SÉchan, La légende d'Hippolyte dans l'Antiquité, in $R E G, 24$ (1911), p. 105 sq.

60 Cf. M. Napoli, Civiltà della Magna Grecia, Rome, 1969, p. 136-146. Il faut souligner que l'acte officiel de fondation ne coïncide pas nécessairement avec l'arrivée des premiers colons. 
les colons de Trézène ${ }^{61}$. Les deux hypothèses permettent d'envisager que le mythe de la résurrection d'Hippolyte par Asclépios y ait été introduit, même à une date antérieure à la réalisation de la tombe « du Plongeur».

D'autre part, il nous ramènerait à une ambiance orphico-pythagoricienne, puisque Hippolyte aurait été membre d'une secte orphique et initié à Éleusis $^{62}$. En effet, les thèmes illustrés dans la sépulture s'inscrivent dans une perspective eschatologique qu'il serait tentant d'associer à ces doctrines si répandues, non seulement en Grande Grèce, mais aussi en Étrurie ${ }^{63}$. La sobriété des peintures et du mobilier funéraire irait dans le même sens. Le banquet collectif masculin était un rituel constitutif et de renforcement des liens d'une communauté. La représentation de l'au-delà comme une fête symposiaque éternelle est évoquée dans la littérature comme appartenant au domaine orphico-pythagoricien («banquet des purs» dans les « îles des bienheureux », localisées dans le Soleil et la Lune). La musique, présente non seulement dans les fresques, mais aussi dans le matériel funéraire sous forme d'une chelys, était considérée comme un moyen de purification de l'âme, dans un souci eschatologique. L'œuf, garant de renaissance, figure dans la cosmogonie et les tabous alimentaires. Le nombre des branches d'arbres, des degrés de la pylé ${ }^{64}$, des cordes des lyres et des personnages, ainsi que la répartition de ceux-ci sur les quatre parois latérales, correspondent à des nombres significatifs. Le plongeur pourrait être une allégorie de l'âme du défunt, qui, libérée de sa prison corporelle, accède à la vie divine en échappant au cycle des renaissances. L'accumulation de ces éléments est troublante et incite à retenir l'hypothèse d'une tombe pythagoricienne à Poseidonia.

Les fresques de la paroi Ouest jouent un rôle important dans la sémantique eschatologique de la tombe. Elles sont l'aboutissement du grand mouvement cosmique. Celui-ci part de la paroi Est, c'est-à-dire de l'échanson, éphèbe en voie d'intégration dans la société des adultes, et du cratère, image

61 Cette version « classique » s'inspire principalement d'Aristote (Politique, V, 2, 10 : les Trézéniens, ayant fondé Sybaris avec les Achéens, furent expulsés en raison d'un trop grand accroissement de la population) et de Strabon (V, 4, 13 : les Sybarites construisirent un teicbos sur le golfe de Poseidonia). Cf. E. Ciaceri, Storia della Magna Grecia, I. La fondazione delle colonie greche e l'ellenizzamento di città nell'Italia antica, Rome, 1966 p. 277-282; M. MEllo, Strabone, V, 4, 13, e le origini di Poseidonia, in PP, 22 (1967), p. 401 sq.; G. Pugliese Carratelli, Per la storia di Poseldonia, in Poseidonia-Paestum. Atti del XXVII Convegno di Studi sulla Magna Grecia, I, Naples, 1988, p. 13-31, particulièrement p. 14-17.

62 Cf. Eur., Hipp., 25-26 et 952-954 (trad. L. Méridier, CUF).

63 Cf. G. Caputo, in Il dibattito, in Atti Taranto VIII, op. cit. (n. 23), p. 264; Napoli, op. cit. (n. 25), p. 165; Somville, loc. cit. (n. 32); Warland, loc. cit. (n. 2).

64 Pour l'identification de ce motif à la borne frontière entre le monde des vivants et le monde des morts, en l'absence de comparaisons iconographiques indiscutables, voir Bianchi Bandineli, loc. cit. (n. 33) 
de mesure et contenant à symbolique matricielle, métaphore de la mer ${ }^{65}$. Sur le couvercle, zone céleste de la sépulture, il se concrétise dans l'envol sinistrogyre du défunt, doublet d'Hélios, qui franchit okéanos et resurgit, couronné, dans le sobre kômos de la paroi Ouest. Comment résister à la tentation d'évoquer les formules orphiques garantes de renaissance : « Mais quand l'âme abandonne la lumière du soleil, à droite [...] Salut, toi qui souffris la passion, que tu n'avais jamais souffert auparavant: d'homme tu devins un dieu; chevreau, tu tombas dans le lait. Salut, salut, toi qui avances vers la droite dans les prés sacrés et les bois de Perséphone " ${ }^{66}$ ?

DaisY WARLAND

Rue Saint-Vincent, 40

B - 4020 LIÈGE

65 L'eau et le vin sont intimement mêlés dans l'expression oinops pontos, depuis l'épopée homérique jusqu'aux poètes lyriques ( $c f$. Lissarrague, op. cit. [n. 8], p. 103). Le breuvage qui confère l'immortalité est obtenu, dans la plupart des mythologies indoeuropéennes, par brassage de la mer, notamment chez les Hindous, les Iraniens, les Scandinaves et les Grecs ( $c f$. G. DumÉziL, Le festin d'immortalité. Étude de mytbologie comparée indo-européenne, Paris, 1924). Les enfants spartiates étaient baignés dans du vin pur dès leur naissance. Les épileptiques mouraient et les nouveau-nés de constitution saine voyaient leur vigueur augmentée (Plut., Vie de Lycurgue, 16, 3). Ce rite d'initiation qui imprègne l'enfant de la force du vin, correspond aux purifications religieuses par l'eau ( $c f$. M. Eliade, Images et symboles. Essai sur le symbolisme magico-religieux, Paris, 1952 , p. 201).

66 Lamelle d'or de Thurii, museo archeologico nazionale de Naples $n^{\circ} 11463$, première moitié du IV ${ }^{\mathrm{e}}$ siècle av. J.-C.; texte original, transcription et reconstitution : $\mathrm{n}^{\circ} \mathrm{A} 4$ de G. Zuntz, Persephone. Three Essays on Religion and Thougbt in Magna Graecia, Oxford, 1971 , p. 328-329. 